\title{
La revisión bibliográfica sistemática en fisiología del ejercicio: recomendaciones prácticas. \\ Literature review in exercise physiology: practical recommendations.
}

\author{
Benito Peinado, Pedro José; Díaz Molina, Víctor; Calderón Montero, Francisco Javier; \\ Peinado Lozano, Ana Belén; Martín Caro, Carlos; \\ Álvarez Sánchez, María; Pérez Tejero, Javier \\ Universidad Politécnica de Madrid
}

\section{Resu m e n}

La revisión bibliográfica no sólo es paso fundamental para el diseño de proyectos de investigación, sino para el entrenador, el docente y el gestor en Ciencias de la Actividad Física y del Deporte que pretenden estar informados en sus respectivas actividades profesionales. Por otro lado, la revisión bibliográfica permite centrar el objetivo y el tema de una investigación, siendo necesaria por humildad científica y para responder a la pregunta "¿Habré buscado en todos los lugares posibles?". Así, el objetivo de este artículo es proporcionar información útil y práctica a aquellas personas que se enfrentan a la revisión de la literatura con diferentes fines, entre los que se pueden encontrar los proyectos de investigación, las tesis doctorales, los artículos de revisión o simplemente la satisfacción personal de conocer profundamente un aspecto de la realidad.

\section{Abstract}

Literature review is not only a key step designing invetigations projects, is fundamental for trainers, teachers a managers in Physical Activity and Sports Sciences who try to be informed about their respective professional activities. In the other hand, literature review perimts center the objetive and the topic of a investigation, being necessary for scientific humility and to respond the cuestion "Will I have looked for in all the possible places?". Thus, the aim of this article is to provide useful and practical information for people who has to face a literature review with diferents porpouses, between which we find investigation projects, doctoral thesis, review articles or the personal satisfaction to know deeply an aspect the reality.

Palabras clave: Revisión, literatura, búsqueda, fisiología del ejercicio, ciencias de la actividad física y del deporte.

Key Words: Review, literatura, search, physiology of exercise, sport sciences.

Correspondencia/correspondence: Pedro José Benito Peinado

Laboratorio de Fisiología de la Facultad de Ciencias de la Actividad Física y del Deporte. INEF. Universidad Politécnica de Madrid.

C/Martín Fierro, S/N 28040 - Madrid - Spain

Telf: 913364070.

E-mail: pedroj.benito@upm.es 
Benito P., P. J.; Diaz M., V.; Calderón M. J.; Peinado L., A.B.; Martín C., C.; Álvarez S., M.; Morencos M., E.; Pérez T., J. (2007). La revisión bibliográfica sistemática en fisiología del ejercicio: recomendaciones prácticas. Revista Internacional de Ciencias del Deporte. 6(3), 1-11. http://www.cafyd.com/REVISTA/art1n6a07.pdf

\section{Introducción}

L a revisión bibliográfica sistemática responde a varias razones. Entre otras es necesaria por humildad científica, ya que reconocer y criticar el trabajo de otro permite el avance del conocimiento científico, además de poder opinar con fundamento de un área específica del saber. Además, la revisión permite centrar el objetivo de la investigación porque ayuda a encontrar cuestiones sin resolver de estudios previos.

Cuando nos enfrentamos al diseño de una investigación debemos seguir una serie de pasos entre los que se encuentra la revisión bibliográfica como uno de los más esforzados y a la vez más trascendentes. Ésta es un paso clave en el diseño, porque debe preceder a la formulación de hipótesis, si queremos que éstas sean adecuadas y coherentes con teorías y conocimientos previos (figura 1). Para formular una pregunta de investigación podemos proceder deductivamente (desde la teoría hacia las cuestiones particulares) o inductivamente (de la falta de resolución de problemas hacia las teorías). La revisión de la literatura, si es exhaustiva, crea una base estable de conocimiento desde la que se puede inducir o deducir una o varias preguntas sobre las que se construya nuestra investigación.

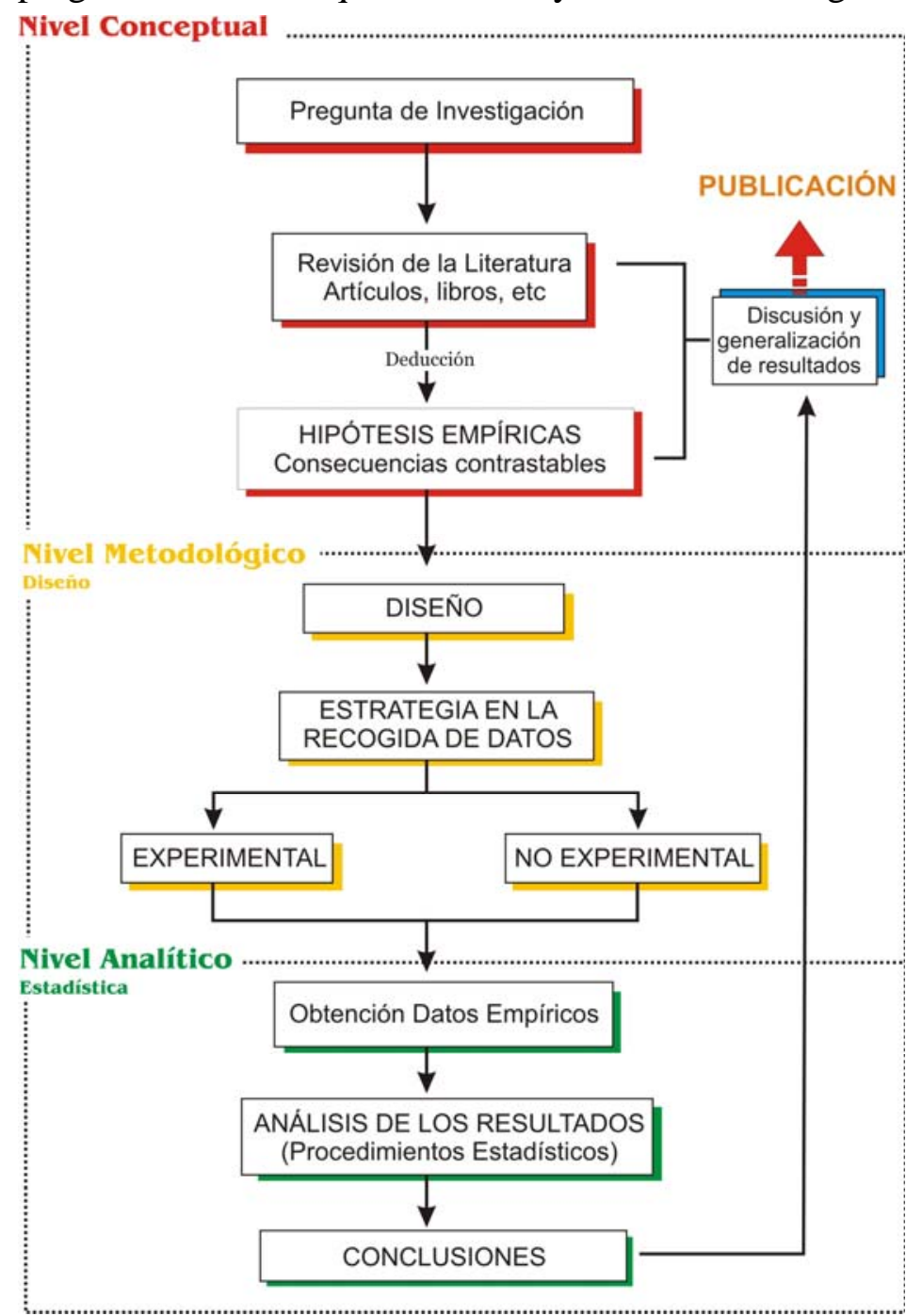

Es necesario destacar que la revisión de la literatura es un paso enmarcado dentro de un proceso mucho más amplio en el método científico, constituido por una serie de fases (figura 1). Dentro de este proceso es necesario cumplir cada paso para seguir avanzando con seguridad, ya que las posibilidades de establecer relaciones de causalidad al finalizar un proyecto de investigación, dependerán del diseño experimental elegido.

Fig 1. Pasos a seguir en la realización de un proyecto de investigación. Modificado de (Doménech, 1999:47).

Además es necesario considerar que aunque casi todos los trabajos sitúan la fase de documentación al inicio del proceso, esto no es del todo cierto, ya que a lo largo de todo el proceso de investigación hay que permanecer alerta sobre la aparición de nuevos documentos relacionados con nuestro trabajo. 
Benito P., P. J.; Diaz M., V.; Calderón M. J.; Peinado L., A.B.; Martín C., C.; Álvarez S., M.; Morencos M., E.; Pérez T., J. (2007). La revisión bibliográfica sistemática en fisiología del ejercicio: recomendaciones prácticas. Revista Internacional de Ciencias del Deporte. 6(3), 1-11. http://www.cafyd.com/REVISTA/art1n6a07.pdf

La importancia de esta fase, radica en poder construir un filtro frente a las fuentes de información científica existentes, evitando el desbordamiento informativo (hecho que crea mucha incertidumbre a los investigadores y disminuye la perdida de tiempo en cuestiones adyacentes a la temática del estudio). Disponer de las herramientas necesarias y adecuadas para buscar, clasificar, ordenar y recuperar información en la era de la "red" no es labor sencilla. Este trabajo intenta ayudar a los investigadores en Ciencias de la Salud y más concretamente en Fisiología del Esfuerzo, a identificar fuentes, conseguir destrezas y clasificar información con el objetivo de responder a una relevante cuestión: ¿He buscado pertinentemente toda la información que puede apoyar o delimitar mis afirmaciones?

Es mucha la literatura escrita o dedicada a mostrar pasos y estrategias a seguir (Creswell, 2003:69; Gratton \& Jones, 2004; Gutiérrez Dávila \& Oña Sicilia, 2005; Haag \& Borms, 2004; Thomas \& Nelson, 2001), aunque en numerosas ocasiones esta información es demasiado general. Pero existen honrosas excepciones. Este es el caso del manuscrito de GutiérrezDávila y Oña, en el que la revisión documental se encuentra a caballo entre la identificación del problema y los datos empíricos (Gutiérrez Dávila \& Oña Sicilia, 2005), dicho de otra forma, es lo que nos aparta de sacar conclusiones, contrastables, verdaderas y relevantes.

De forma más concreta, el objetivo de este artículo es establecer una serie de pasos dentro de la revisión bibliográfica, para ofrecer información útil y práctica a aquellas personas que se enfrenten a la realización de una revisión, con vistas a diseñar un proyecto, o bien con el objetivo de obtener información.

En numerosas ocasiones estas búsquedas sistemáticas de algunos investigadores se convierten en "meta-análisis", que consisten fundamentalmente en interpretar un conjunto de resultados de estudios realizados en condiciones similares (diseños experimentales parecidos). Este procedimiento presenta ventajas e inconvenientes que no son motivo de nuestro trabajo, pero pueden ser ampliados notablemente en los trabajos de Egger y Smith (Egger \& Smith, 1997; Egger, Smith, \& Phillips, 1997).

\section{Paso 1: Elección de las palabras clave y elaboración de la frase de búsqueda}

Antes de comenzar a buscar es necesario tener claro el tema de trabajo, de forma que, podamos escoger una serie de palabras que llamaremos palabras clave. Éstas son definidas por la Real Academia de la Lengua Española como "de entre las palabras que forman un título o entran en un documento, las más significativas o informativas sobre su contenido.” (RAE, 2001). Las palabras clave se relacionan entre sí a través de conectores lógicos (tabla 1). Estos conectores provienen del álgebra de Bool (Hortalá, 2001:344) y se les puede aplicar leyes matemáticas simples como la ley asociativa y la ley distributiva. 
Benito P., P. J.; Diaz M., V.; Calderón M. J.; Peinado L., A.B.; Martín C., C.; Álvarez S., M.; Morencos M., E.; Pérez T., J. (2007). La revisión bibliográfica sistemática en fisiología del ejercicio: recomendaciones prácticas. Revista Internacional de Ciencias del Deporte. 6(3), 1-11. http://www.cafyd.com/REVISTA/art1n6a07.pdf

\begin{tabular}{|c|l|}
\hline Conector & \multicolumn{1}{c|}{ Significado } \\
\hline Y - AND & $\begin{array}{l}\text { Si relacionamos dos palabras con este conector, la base de datos nos devuelve } \\
\text { los documentos en los que aparecen únicamente ambas palabras a la vez. }\end{array}$ \\
\hline O - OR & $\begin{array}{l}\text { Si relacionamos dos palabras con este conector, la base de datos devuelve los } \\
\text { documentos en los que aparecen ambas palabras o bien una de las dos. }\end{array}$ \\
\hline NO - NOT & $\begin{array}{l}\text { Este conector poco habitual no relaciona dos palabras, pero puede preceder a } \\
\text { una en concreto, permitiendo que la base de datos no devuelva documentos que } \\
\text { contengan esa palabra. }\end{array}$ \\
\hline
\end{tabular}

Tabla 1: Conectores universalmente utilizados y su significado.

Por lo tanto, para encontrar información sobre un tema, tendremos que relacionar las palabras clave mediante los conectores con el fin de elaborar una frase de búsqueda, que podría ser definida como el "conjunto con sentido de palabras clave relacionadas mediante conectores lógicos”.

Un aspecto a tener en cuenta a la hora de formar una frase de búsqueda es la utilización de los paréntesis y/o caracteres comodines. El más frecuente (aunque no el único) es el asterisco “*”, que indica la raíz y cualquier continuación. Normalmente, el operador AND tiene prioridad sobre la operación realizada por el operador OR; sin embargo, recomendamos utilizar paréntesis para no tener dudas sobre lo que estamos buscando. En el ejemplo que planteamos a continuación pueden verse con claridad distintas posibilidades.

Supongamos que nuestras palabras clave son heart rate, exercise y physical activit*.

Podremos formar las siguientes frases:

(heart rate AND exercise) OR physical activity = (heart rate OR exercise) AND (exercise OR physical activit*) (frase 1)

alternando el orden de los paréntesis

heart rate AND (exercise OR physical activity) = (heart rate AND exercise) OR (heart rate AND physical activit*) (frase 2)

La frase 1, devuelve los documentos que contienen alguna de las tres palabras, mientras que la frase 2, más restrictiva, incluye siempre la palabra heart rate, bien con exercise, bien con physical activity o activities, o cualquier raíz que contenga "activit” seguido de cualquier cosa.

La elección de las palabras clave y elaboración de la frase de búsqueda es, por otro lado, de vital importancia en la búsqueda bibliográfica, y de este paso dependerá encontrar los documentos que estamos buscando. Nosotros recomendamos las siguientes estrategias: 
Benito P., P. J.; Diaz M., V.; Calderón M. J.; Peinado L., A.B.; Martín C., C.; Álvarez S., M.; Morencos M., E.; Pérez T., J. (2007). La revisión bibliográfica sistemática en fisiología del ejercicio: recomendaciones prácticas. Revista Internacional de Ciencias del Deporte. 6(3), 1-11. http://www.cafyd.com/REVISTA/art1n6a07.pdf

1. Utilizar los tesauros para encontrar palabras clave. Los tesauros son diccionarios de sinónimos creados por documentalistas para clasificar artículos o documentos. Los tesauros pueden encontrarse en la mayoría de las bases de datos y suelen ir acompañados de un tutorial explicando su funcionamiento (ERL WebSPIRS, 2006b; National Center of Biotechnology Information, 2006a).

2. Tomar palabras clave de documentos que se hayan leído previamente y estén relacionados con el tema del estudio.

3. Preguntar a algún experto en el tema para que nos oriente sobre las palabras clave que podemos utilizar.

4. Agrupar las palabras clave en categorías. De esta forma, las palabras que se encuentren en la misma categoría irán encerradas entre paréntesis y conectadas por OR, mientras que cada grupo de palabras irá relacionados con AND. En la tabla 2 se muestra un ejemplo de agrupación cuando queremos saber qué gasto energético tiene un entrenamiento con cargas en personas jóvenes.

\begin{tabular}{|c|c|c|c|}
\hline Categoría & Entrenamiento & Gasto energético & Población \\
\hline $\begin{array}{c}\text { Palabras clave } \\
\text { relacionadas }\end{array}$ & $\begin{array}{c}\text { Resistance training } \\
\text { strength } \\
\text { power } \\
\text { circuit training }\end{array}$ & $\begin{array}{c}\text { Energy expenditure } \\
\text { Oxygen } \\
\text { consumption }\end{array}$ & Elder* \\
\hline
\end{tabular}

Tabla 2: Categorías y agrupación de las palabras clave, para la formación de la frase final.

La frase de búsqueda quedaría, por lo tanto, de la siguiente manera:

(Resistance training OR strength OR power OR circuit training) AND (energy expenditure OR oxygen consumption) AND NOT (elder*)

El motivo de incluir el asterisco en la frase es la de que no incluya ningún sujeto mayor, es decir artículos que contengan el término elderly, elder, etc.

No debemos perder de vista que el objetivo final de la generación de una frase de búsqueda es poder incluirla en cuantas bases de datos podamos y sean relevantes para nuestra investigación. Existen varias estrategias con el fin de reducir el número de documentos que nos devolverá una base de datos. En numerosas ocasiones, podemos limitar la búsqueda a los documentos publicados en los últimos cinco años o especificar que la frase de búsqueda aparezca únicamente en el resumen o el título. De esta forma, tendremos un número razonable de documentos que revisar para decidir cuáles de ellos tenemos que conseguir a texto completo.

\section{Paso 2: Búsqueda de información en libros y tesis doctorales}

Este paso puede ser un buen comienzo para empezar a leer e informarse sobre lo investigado hasta el momento en un tema en concreto. La búsqueda de información en libros se realiza de la forma habitual, es decir, introduciendo las palabras clave o la frase de búsqueda en los ordenadores de una biblioteca, que puede estar especializada, como es el caso de las facultades y escuelas universitarias, o ser de carácter general (UPM, 2003). 
Benito P., P. J.; Diaz M., V.; Calderón M. J.; Peinado L., A.B.; Martín C., C.; Álvarez S., M.; Morencos M., E.; Pérez T., J. (2007). La revisión bibliográfica sistemática en fisiología del ejercicio: recomendaciones prácticas. Revista Internacional de Ciencias del Deporte. 6(3), 1-11. http://www.cafyd.com/REVISTA/art1n6a07.pdf

La búsqueda de información en tesis doctorales es algo más compleja, ya que no se trata de publicaciones de tipo divulgativo. Existen algunos buscadores de tesis doctorales que se muestran en la tabla 3.

\begin{tabular}{|l|l|}
\hline \multicolumn{1}{|c|}{ URL } & \multicolumn{1}{c|}{ Descripción } \\
\hline http://www.mcu.es/TESEO/teseo.html & $\begin{array}{l}\text { Es una base de datos del Ministerio de } \\
\text { Educación y Ciencia. Permite buscar tesis y } \\
\text { conocer su ubicación en las universidades } \\
\text { españolas. }\end{array}$ \\
\hline http://wwwlib.umi.com/dissertations/search & $\begin{array}{l}\text { Es una base de datos con un gran número } \\
\text { de títulos de universidades americanas y } \\
\text { canadienses. Algunas tesis se pueden } \\
\text { comprar y en algunos casos está permitido } \\
\text { imprimir las 24 primeras páginas. Para } \\
\text { consultar la base de datos completa es } \\
\text { necesario acceder desde una institución, } \\
\text { como por ejemplo, una universidad. }\end{array}$ \\
\hline http://dialnet.unirioja.es/ & $\begin{array}{l}\text { Contiene artículos en castellano y tesis } \\
\text { doctorales, algunas en acceso completo. }\end{array}$ \\
\hline http://www.cervantesvirtual.com/tesis/tesis_catalogo.shtml & $\begin{array}{l}\text { Esta base de datos incluye un buen número } \\
\text { de tesis doctorales de varias universidades. } \\
\text { Pueden consultarse a texto completo. Es } \\
\text { necesario respetar los derechos de autor. }\end{array}$ \\
\hline http://www.cafyd.com & $\begin{array}{l}\text { Es uno de los portales en Ciencias de la } \\
\text { Actividad Física y del Deporte más } \\
\text { interesantes y que además contiene entre } \\
\text { sus colaboradores la mayor parte de las } \\
\text { tesis doctorales en esta disciplina. }\end{array}$ \\
\hline
\end{tabular}

Tabla 3: Direcciones web para encontrar tesis doctorales

Normalmente, es necesario conocer al autor o el título de la tesis que buscamos, siendo a veces muy complicado encontrar alguna referencia. En cualquier caso, recomendamos no dejar de intentarlo, ya que nuestro trabajo puede no ser original.

\section{Paso 3: Búsqueda de información en artículos}

Los artículos de revistas científicas especializadas son seguramente la fuente de información más utilizada y permiten tener ésta muy actualizada. Si las palabras clave están bien elegidas y la frase de búsqueda bien redactada, no tendremos problema en encontrar artículos que documenten estudios previos sobre nuestro tema de interés.

Existen varias bases de datos en las que se pueden encontrar referencias bibliográficas y artículos. En español, podemos acceder al Instituto de la Salud Carlos III (Ministerio de Sanidad y Consumo, 2006b), que ofrece en sus servicios el acceso a Scielo (Scientific electronic library online) y a la Biblioteca Virtual de la Salud (Ministerio de Sanidad y Consumo, 2006a; SCIELO España, 2006). En estos dos sitios web podemos encontrar artículos en español a texto completo de varias revistas relacionadas con la medicina y la salud. Además, en el segundo de estos recursos electrónicos, podemos acceder si pinchamos 
Benito P., P. J.; Diaz M., V.; Calderón M. J.; Peinado L., A.B.; Martín C., C.; Álvarez S., M.; Morencos M., E.; Pérez T., J. (2007). La revisión bibliográfica sistemática en fisiología del ejercicio: recomendaciones prácticas. Revista Internacional de Ciencias del Deporte. 6(3), 1-11. http://www.cafyd.com/REVISTA/art1n6a07.pdf

en Búsqueda en bases de datos a otras bases de datos nacionales e internacionales, así como a catálogos colectivos entre los que destaca C17 ó Catálogo Colectivo de Publicaciones Periódicas de las Bibliotecas de Ciencias de la Salud Españolas (Ministerio de Sanidad y Consumo, 2006c). Se puede completar la búsqueda de artículos en castellano consultando la Coordinadora de Documentació Biomèdica (Coordinadora de Documentació Biomèdica, 2006), el Catálogo Colectivo de Publicaciones Periódicas de la Biblioteca Nacional (CCPP) (Biblioteca Nacional de España, 2006) y el servicio DIALNET de la Universidad de la Rioja (Universidad de La Rioja, 2006). Otra fuente de datos que nos parece bastante relevante, aunque contiene información fundamentalmente de artículos en castellano es la Base de Datos Redalyc que es considerada la hemeroteca en línea de artículos en nuestra lengua (Universidad Autónoma del Estado de México, 2006). Estas dos últimas son la única alternativa a escudriñar uno por uno los resúmenes de cada revista en nuestra biblioteca especializada.

Si nos disponemos a buscar bibliografía en inglés (principalmente), tendremos que utilizar las dos bases de datos más importantes en Fisiología del Ejercicio y Deportes. Éstas son PUBMED y SPORT DISCUS. El sistema de búsqueda PubMed es un proyecto desarrollado por la National Center for Biotechnology Information (NCBI) en la National Library of Medicine (NLM) que permite la búsqueda entre 15 millones de referencias, es de acceso gratuito y publico (National Center of Biotechnology Information, 2006b). Sport Discus es una Base de datos bibliográfica de pago, creada por el Sport Information Resource Centre (SIRC) de Canadá y que contiene información sobre: medicina del deporte, fisiología del ejercicio, biomecánica, psicología, técnicas de preparación física, entrenamiento, educación física, condición física, vida activa, recreación, historia, instalaciones y equipamiento. Incluye citas de más de 47.500 libros, actas de conferencias, tesis, informes, y otras monografías; artículos indexados de más de 2.000 publicaciones periódicas, revistas y boletines actuales y cada año se añaden más de 26.000 documentos nuevos (ERL WebSPIRS, 2006a). Su principal problema proviene de la heterogeneidad en su contenido, aunque algunos investigadores observan en ésto una virtud para nosotros dudosa. En resumen, ambas son muy potentes, contienen un gran número de títulos y referencias, y lo que es más importante, poseen un tesauro (ERL WebSPIRS, 2006b; National Center of Biotechnology Information, 2006a) que facilita la búsqueda y existen tutoriales online a cerca de su funcionamiento y utilización.

Normalmente, todas las bases de datos, permiten restringir la búsqueda a una serie de apartados, es decir, podemos introducir nuestra frase de búsqueda y pedir que sólo examine el título o el resumen del artículo. Esto suele ser muy útil para restringir adecuadamente la búsqueda evitando la aparición de un gran número de artículos que pueden ser potencialmente no deseables.

Es necesario subrayar que en ocasiones es necesario acceder a estas bases de datos desde una institución como puede ser la universidad (Universidad de La Rioja, 2006; UPM, 2003). Normalmente, las bibliotecas especializadas poseen acceso a diferentes bases de datos de publicaciones periódicas, de modo que no hay que dejar de pedir ayuda al responsable para evitar pérdida de información, o el acceso más rápido a la misma. 
Benito P., P. J.; Diaz M., V.; Calderón M. J.; Peinado L., A.B.; Martín C., C.; Álvarez S., M.; Morencos M., E.; Pérez T., J. (2007). La revisión bibliográfica sistemática en fisiología del ejercicio: recomendaciones prácticas. Revista Internacional de Ciencias del Deporte. 6(3), 1-11. http://www.cafyd.com/REVISTA/art1n6a07.pdf

\section{Paso 4: Acceso a las revistas y adquisición de artículos}

Para acceder a las revistas y obtener los artículos a texto completo tendremos que utilizar los recursos electrónicos nombrados anteriormente, siempre y cuando permitan el acceso a los artículos, o bien, visitar una biblioteca. La Red de Bibliotecas Universitarias, alojada en la Conferencia de Rectores de Universidades Españolas (CRUE), permite buscar en el catálogo de publicaciones periódicas las revistas y su ubicación dentro de bibliotecas universitarias y de hospitales españoles, de modo que, es una buena forma de localizar revistas (CRUE, 2006).

La adquisición de los artículos es la parte final de esta búsqueda. La copia legal de la totalidad o parte de los originales se suele resolver en las direcciones web o en las bibliotecas concretas. La búsqueda final no debe exceder del centenar de trabajos y se recomienda comenzar por las revisiones, como medio de retroalimentar el proceso de construcción de la frase de búsqueda. Algunos trabajos hablan de que en las primeras búsquedas el número de trabajos que debemos adquirir no debe superar las 50 unidades (Creswell, 2003:34), ya que un número superior puede ser desbordante y hacer inaccesible su tratamiento.

Además de esto, es necesario permanecer al día sobre las nuevas publicaciones relacionadas con nuestro tema. Es decir una vez que hemos acabado la búsqueda a una fecha determinada, ¿qué hecho impide que en el próximo número de cualquier revista revisada incluya un nuevo trabajo que podría interesarnos?, ¿Cómo podemos entonces estar alerta de nuevas publicaciones? Casi todas las bases de datos tienen un servicio de envío por correo electrónico de resúmenes a partir de palabras clave o temas concretos. Para esto, es posible registrarse en ISI web of knowledge o en las alertas de PUBMED y Sport Discus (ERL WebSPIRS, 2006a; National Center of Biotechnology Information, 2006b; Thomson Corporation, 2006). La segunda opción es incluir de nuevo la frase en las bases de datos, buscando a partir de la fecha en que cerramos la última búsqueda.

\section{Paso 5: Tratamiento de la información}

A medida que avancemos en la búsqueda iremos almacenando documentos. Del proceso de almacenamiento y tratamiento de la información de estos documentos dependerá que los podamos utilizar en un futuro de forma rápida y adecuada.

En primer lugar, recomendamos hacer una ficha resumen (digital o manuscrita) de cada texto, en la que se incluyan campos como la referencia bibliográfica completa, el objetivo del estudio, las características de la muestra, el diseño experimental, el análisis estadístico realizado, los resultados más importantes, las conclusiones y las posibles limitaciones del estudio, entre otros. Esta ficha se puede almacenar con su respectivo documento y numerar ambos con el fin de generar una base de datos de documentos que puede crearse en programas como Endnote ${ }^{\circledR}$, Procite $\AA$, Referente Manager $\AA$, Biblioscape ${ }^{\circledR}$ capaz de almacenar referencias e introducirlas en textos con varias normativas. Estos gestores de referencias bibliográficas son imprescindibles hoy en día para el manejo razonable de la información.

En segundo lugar, puede ser muy útil elaborar un árbol de conocimiento o mapa de la literatura como el propuesto por Creswell (Creswell, 2003:39). En este gráfico en forma de 
Benito P., P. J.; Diaz M., V.; Calderón M. J.; Peinado L., A.B.; Martín C., C.; Álvarez S., M.; Morencos M., E.; Pérez T., J. (2007). La revisión bibliográfica sistemática en fisiología del ejercicio: recomendaciones prácticas. Revista Internacional de Ciencias del Deporte. 6(3), 1-11. http://www.cafyd.com/REVISTA/art1n6a07.pdf

árbol (figura 2), cada rama es un tema y contiene los artículos que tratan sobre el mismo. Estos gráficos son realmente útiles a la hora de escribir un artículo o tesis, ya que, al clasificar los artículos por temas, podemos saber rápidamente que documentos debemos utilizar en cada sección del artículo o tesis.

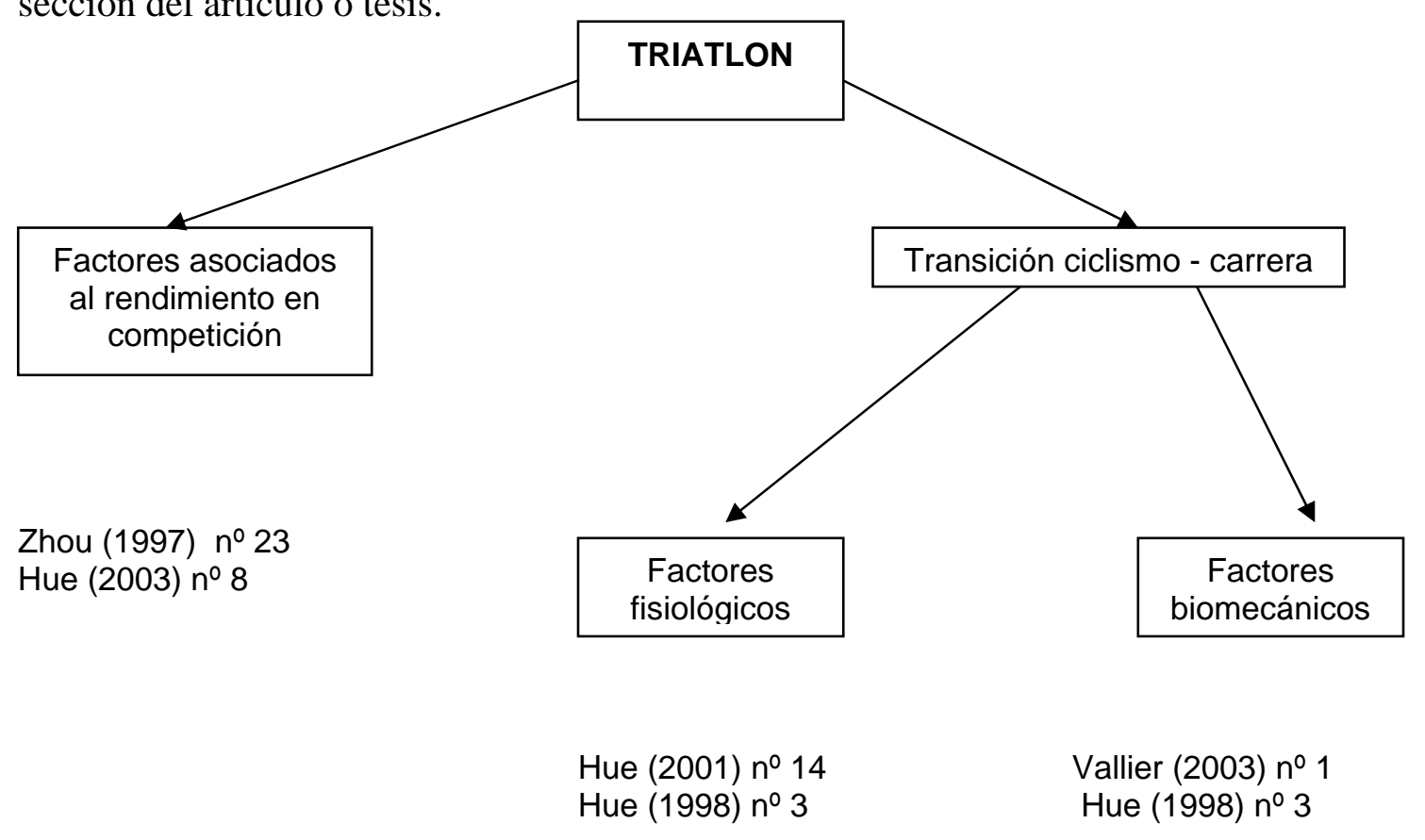

Fig. 2. Gráfico en forma de árbol para clasificar los documentos relativos a un estudio sobre la segunda transición en el triatlón.

Una alternativa muy plausible y más operativa según nuestro criterio (aunque consideramos que no es excluyente, se pueden hacer ambas) es construir un índice temático como el propuesto muy acertadamente por Umberto Eco. Consiste en crear un índice a priori e ir introduciendo en cada epígrafe con qué documento pensamos desarrollarlo (Eco, 2001:146). Siguiendo con el ejemplo anterior, la tabla 4 muestra los resultados del hipotético índice:

\begin{tabular}{|c|l|}
\hline \multirow{2}{*}{$\begin{array}{c}\text { 1. Introducción } \\
\text { 1.1 Factores asociados al rendimiento en } \\
\text { competición }\end{array}$} & $\begin{array}{l}\text { Zhou }(1997) \mathrm{n}^{\circ} 23 ; \text { Hue }(2003) \mathrm{n}^{\circ} \\
\text { 8; Hue }(2001) \mathrm{n}^{\circ} 14 ; \\
\text { Vallier }(2003) \mathrm{n}^{\circ} 1 .\end{array}$ \\
\hline $\begin{array}{c}\text { Zhou }(1997) \mathrm{n}^{\circ} 23 . \\
\text { Hue }(2003) \mathrm{n}^{\circ} 8 .\end{array}$ \\
\hline 1.1.1 Factores fisiológicos & $\begin{array}{l}\text { Hue }(2001) \mathrm{n}^{\circ} 14 . \\
\text { Hue }(1998) \mathrm{n}^{\circ} 3 .\end{array}$ \\
\hline 1.1.1 Factores biomecánicos & $\begin{array}{l}\text { Vallier }(2003) \mathrm{n}^{\circ} 1 . \\
\text { Hue }(1998) \mathrm{n}^{\circ} 3 .\end{array}$ \\
\hline
\end{tabular}

Tabla 4: Índice temático aplicando el procedimiento de Eco, 2001. 
Benito P., P. J.; Diaz M., V.; Calderón M. J.; Peinado L., A.B.; Martín C., C.; Álvarez S., M.; Morencos M., E.; Pérez T., J. (2007). La revisión bibliográfica sistemática en fisiología del ejercicio: recomendaciones prácticas. Revista Internacional de Ciencias del Deporte. 6(3), 1-11. http://www.cafyd.com/REVISTA/art1n6a07.pdf

\section{Conclusiones}

La revisión bibliográfica se enmarca dentro de un proceso de investigación mucho más amplio como mencionábamos anteriormente; sin embargo, por sus características es de vital importancia para que un proyecto se construya con una base adecuada. Además, la revisión es en sí un proceso continuo que se retroalimenta (figura 3) y no termina. Además existe una importancia añadida en la profesionalización de cualquier sector de formación, especialmente en la formación de profesores universitarios y dentro de ellos los que más nos interesan, los especialistas en Ciencias de la Actividad Física y del Deporte, que debido a lo incipiente de nuestra ciencia, tenemos que demostrar con mayor ahínco nuestro rigor científico. Pero sobre todo, el éxito de una buena revisión bibliográfica es planificarla adecuadamente (Thomas \& Nelson, 2001:48).

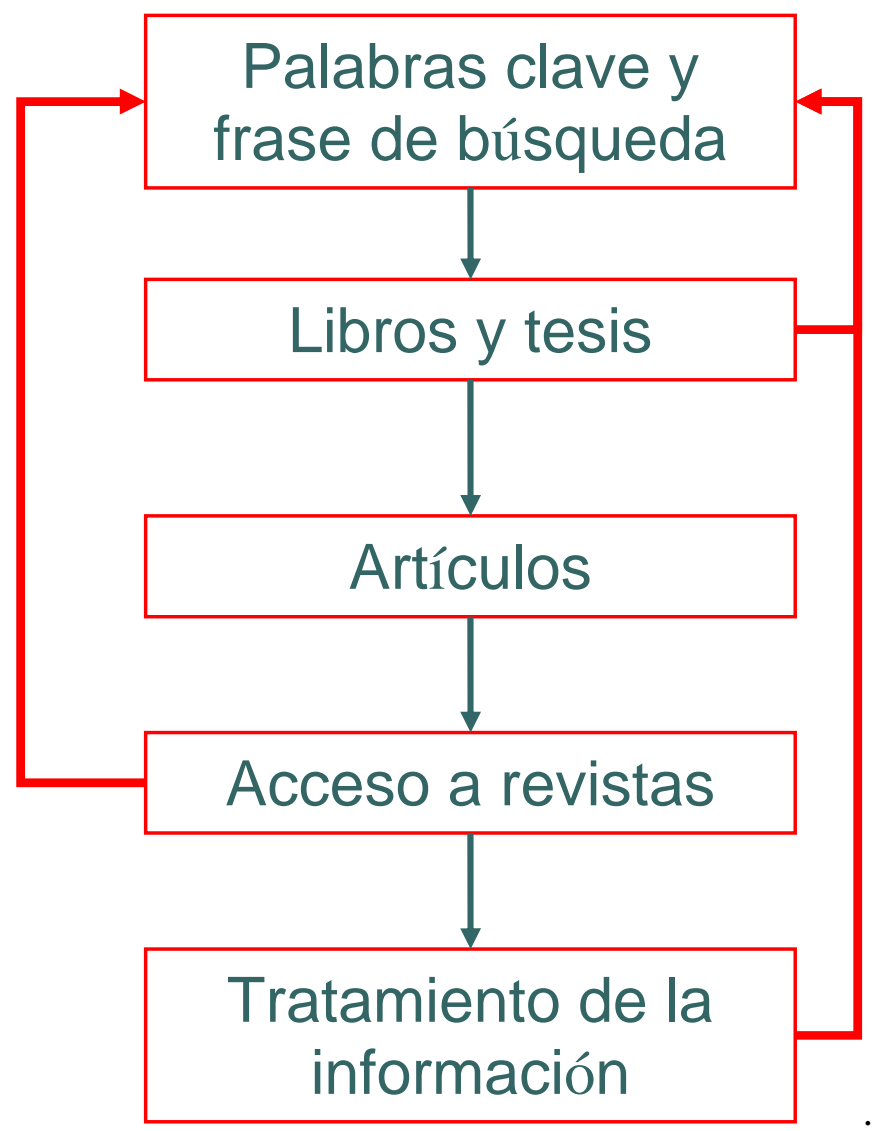

Fig. 3. Esquema representativo del proceso de búsqueda bibliográfica. 
Benito P., P. J.; Diaz M., V.; Calderón M. J.; Peinado L., A.B.; Martín C., C.; Álvarez S., M.; Morencos M., E.; Pérez T., J. (2007). La revisión bibliográfica sistemática en fisiología del ejercicio: recomendaciones prácticas. Revista Internacional de Ciencias del Deporte. 6(3), 1-11. http://www.cafyd.com/REVISTA/art1n6a07.pdf

\section{Referencias bibliográficas}

Biblioteca Nacional de España. (2006, 28/06/06). Catálogo Colectivo de Publicaciones Periódicas. Retrieved 28/06/06, 2006, from: http://www. bne.es/cgi-bin/wsirtex?FOR=WBNCCPP4

Coordinadora de Documentación Biomédica. (2006, 28/06/06). Catálogo de la Coordinadora de Documentación Biomédica. Retrieved 28/06/2006, 2006, from: http://www.doc6.es/cdb/

Creswell, J. W. (2003). Research design: qualitative, quantitative, and mixed method approaches ( 2 a ed.). London: SAGE.

CRUE. (2006, 28/06/06). REBIUN. Retrieved 28/06/06, 2006, from: http://rebiun.crue.org

Doménech, J. M. (1999). Métodos estadísticos en Ciencias de la Salud. Barcelona: Signo.

Eco, U. (2001). Cómo se hace una tesis: técnicas y procedimientos de estudio, investigación y escritura ( $3^{a}$ ed.). Barcelona: Gedisa.

Egger, M., \& Smith, G. D. (1997). Meta-Analysis. Potentials and promise. Bmj, 315(7119), 1371-1374.

Egger, M., Smith, G. D., \& Phillips, A. N. (1997). Meta-analysis: principles and procedures. Bmj, 315(7121), 1533-1537.

ERL WebSPIRS. (2006a, 28/06/06). SPORT Discus. Retrieved 28/06/06, 2006, from: http: //web5s. silverplatter. com/webspirs/start. ws?customer $=c 148207 \&$ language $=e s$

ERL WebSPIRS. (2006b, 28/06/06). Tesauro SPORT Discus. Retrieved 28/06/06, 2006, from: http://web5s. silverplatter.com/webspirs/start.ws?customer $=c 148207 \& l a n g u a g e=e s$

Gratton, C., \& Jones, I. (2004). Research methods for sport studies. London: Routledge.

Gutiérrez Dávila, M., \& Oña Sicilia, A. (2005). Metodología en las ciencias del deporte. Madrid: Síntesis.

Haag, H., \& Borms, J. (2004). Research methodology for sport and exercise science : a comprehensive introduction for study and research: Karl Hofmann.

Hortalá, M. T. (2001). Matemática discreta y lógica matemática (2ae ed.). Madrid: Editorial Complutense.

Ministerio de Sanidad y Consumo. (2006a, 28/06/06). Biblioteca Virtual de la Salud. Retrieved 28/06/06, 2006, from: http://bvs. isciii.es/E/index.php

Ministerio de Sanidad y Consumo. (2006b, 28/06/06). Instituto de la Salud Carlos III. Retrieved 28/06/06, 2006, from: http://www.isciii.es/htdocs/index.jsp

Ministerio de Sanidad y Consumo. (2006c, 28/06/06). Portal C-17. Retrieved 28/06/06, 2006, from: http://isciii.c17.net/index.php

National Center of Biotechnology Information. (2006a, 28/06/06). MeSH. Retrieved 28/06/06, 2006, from http://www.ncbi.nlm.nih.gov/entrez/query.fcgi?db=mesh

National Center of Biotechnology Information. (2006b, 28/06/06). PubMed. Retrieved 28/06/06, 2006, from: http://www.ncbi.nlm.nih.gov/entrez/query.fcgi?DB=pubmed

RAE. (2001, 28/07/03). Diccionario de la Real Academia de la Lengua. Retrieved 28/07/03, 2002, from: http://www.rae.es

SCIELO España. (2006, 28/06/06). Scientific Electronic Library Online. Retrieved 28/06/06, 2006, from: http://wwwscielo.isciii.es/scielo.php 
Benito P., P. J.; Diaz M., V.; Calderón M. J.; Peinado L., A.B.; Martín C., C.; Álvarez S., M.; Morencos M., E.; Pérez T., J. (2007). La revisión bibliográfica sistemática en fisiología del ejercicio: recomendaciones prácticas. Revista Internacional de Ciencias del Deporte. 6(3), 1-11. http://www.cafyd.com/REVISTA/art1n6a07.pdf

Thomas, J. R., \& Nelson, J. K. (2001). Research methods in physical activity (4⿳亠口了 ed.). Campaing, II.: Human Kinetics.

Thomson Corporation. (2006, 28/06/06). ISI Web of knowledge. Retrieved 28/06/06, 2006, from: http://www.accesowok. fecyt.es/login/

Universidad Autónoma del Estado de México. (2006, 28/06/06). Red de Revistas Científicas de América Latina y el Caribe, España y Portugal (REDALYC). Retrieved 28/06/06, 2006, from: http://www.redalyc.com/

Universidad de La Rioja. (2006, 28/06/06). DIALNET. Retrieved 28/06/06, 2006, from: http:// dialnet. unirioja.es/

UPM. (2003, 28/06/06). Catálogo de la Biblioteca de la UPM. Retrieved 28/06/06, from: http://www.upm.es/servicios/bibliotecas/catalogo/catalogo. html 